\title{
Novel Outside-Facility Renovation Technology to Improve Cost-Effectiveness by Long-Term Safe Use
}

\author{
Masaki Waki ${ }^{1, a,{ }^{*}}$, Soichi Ishikawa ${ }^{1, b}$, Gen Kobayashi ${ }^{1, \mathrm{c}}$, Ryouichi Kaneko $^{1, \mathrm{~d}}$, and \\ Hiroaki Tanioka ${ }^{1, e}$
}

${ }^{1}$ NTT Access Network Service Systems Laboratories, 1-7-1 Hanabatake Tsukuba-shi Ibaraki pref., Japan

amasaki.waki.me@hco.ntt.co.jp, bsouichi.ishikawa.ck@hco.ntt.co.jp mail, cgen.kobayashi.wn@hco.ntt.co.jp, dryouichi.kaneko.kv@hco.ntt.co.jp, ehiroaki.tanioka.nh@hco.ntt.co.jp

\section{Keywords: Utility Pole, Telecommunication, FTTH, Outside-Facility}

\begin{abstract}
In order to provide telecommunication and FTTH services, we, NTT, have installed a large number of facilities such as utility poles and optical cables. The number of poles is about 11.9 million and the total length of all installed cables is now about 2.3 million $\mathrm{km}$. These facilities are inspected and maintained by visual inspection by workers every 5 to 10 years, which imposes great costs on the operator. Therefore, we are researching a novel outside-facility renovation technology that can improve cost-effectiveness by long-term safe use. This technology consists of two techniques: a visualization technique of unbalanced tension and a quantitative analysis technique to determine the relationship between unbalanced tension and structural deterioration. In this paper, we describe the concept of the proposed technology. When a utility pole is newly installed, its design assumes that the maximum load consists of wind pressure on the cables. However, when it is impossible to construct a guy wire for bearing the load applied to the utility pole, an unbalanced load occurs because the load cannot be balanced. In addition, when the number of users of various services increases and cables are newly laid, unbalanced loads are generated. Utility poles carrying these unbalanced loads are at significant risk of collapse due to the presence of deflection, inclination, and cracks. Our proposal focuses on the unbalanced load itself, and by detecting and countering it, we aim to enable the use of outside-facilities for a longer period than at present and to reduce replacement costs without sacrificing safety and security. In addition, we also describe how ensuring the long-term safe use of outside-facilities can improve cost-effectiveness. The proposed technique first acquires 3D point cloud data by using 3D laser scanner. It then creates a 3D facility model and calculates the tension in utility poles and cables. In addition, we introduce a novel method to estimate the loads and tension of a whole span from part of the span. Experiments are conducted to compare the estimated and measured values. The results confirm the good agreement of the values (within 10\%) which validates the proposal. We aim to realize a tension visualization scheme with improved accuracy.
\end{abstract}

\section{Introduction}

To provide telecommunication and FTTH services, NTT has installed a large number of facilities such as utility poles and optical cables. Fig. 1 shows an example of the configuration of optical communication equipment in NTT's access network. In addition to optical cables serving as communication paths, NTT is responsible for various outdoor facilities such as utility poles for laying optical cables on the ground, cable tunnels for laying cable underground, and closures for connecting optical cables. There are about 11.8 million poles and a total length of about two 
million $\mathrm{km}$ of cable has been installed [1]. These facilities are mainly maintained and inspected visually at great expense by on-site maintenance staff every 5 to 10 years.

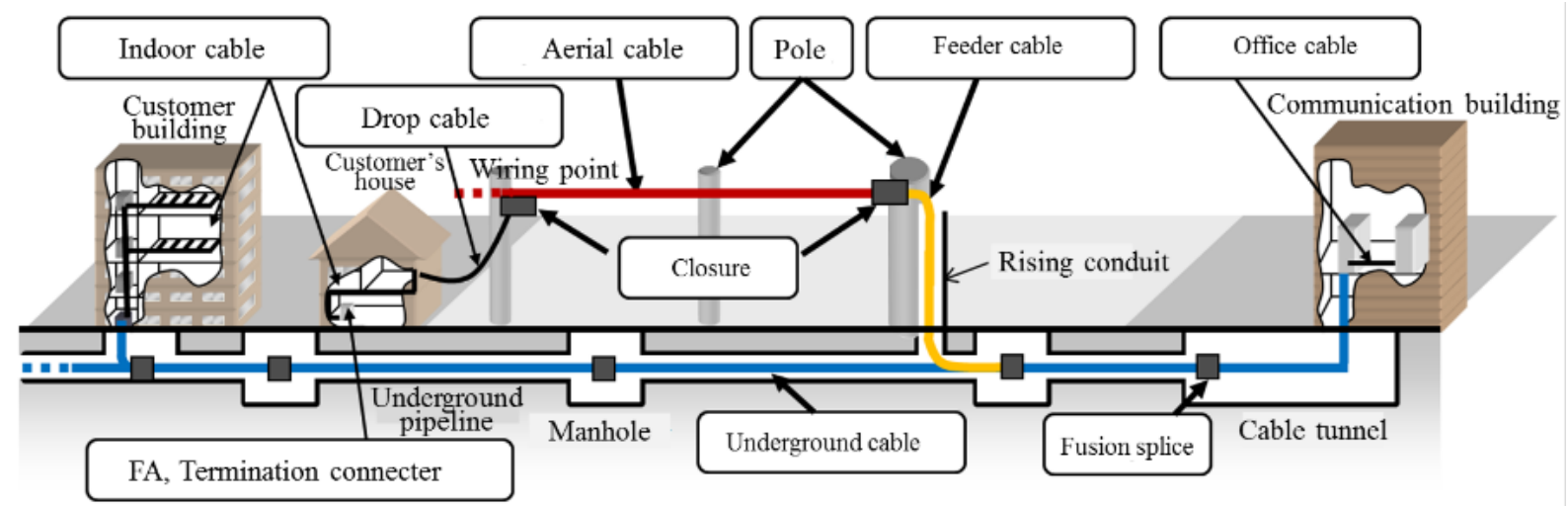

Fig. 1 Outside facilities for FTTH

To date, NTT has been using MMS (Mobile Mapping System) equipped with a laser scanner; 3D models of utility poles were obtained from 3D point groups gathered while driving on the road. Metrics of structural deterioration such as inclination and deflection on a desk were examined and put into practical use to enhance inspection operations [2-4].

In order to achieve long-term safe use of the facilities, this study focuses on the mechanisms of structural deterioration, preventing new deterioration factors from occurring in the future, and alleviating the partial degradation that has already occurred to maintain a "healthy condition" without any structural risk. In addition, the system will replace inspection workers, which is an issue in the current inspection process, and enable easy and accurate health monitoring at the same time.

Fig. 2 shows the process of structural deterioration. This figure shows that the utility poles were structurally sound as designed and constructed, but structural deterioration occurred due to various factors when installed. The various factors are "Influence of attaching materials", which is the increase in load caused by the addition of attachments due to the increase in customers, and "Influence of complex and diverse loads and tension", which is the increase in load imposed by other poles, "The gap between design and actual facilities", the difference between design and actual equipment, and "Natural environment", due to increased wind speeds and disasters. In the current inspection system, inspection workers visually check the utility poles one by one and decide whether to renew or tag for continuous observation according to the degree of structural deterioration such as deflection and inclination. However, manually inspecting the numerous poles every 5 to 10 years is extremely cost-ineffective, as a lot of manpower and labor is required. 


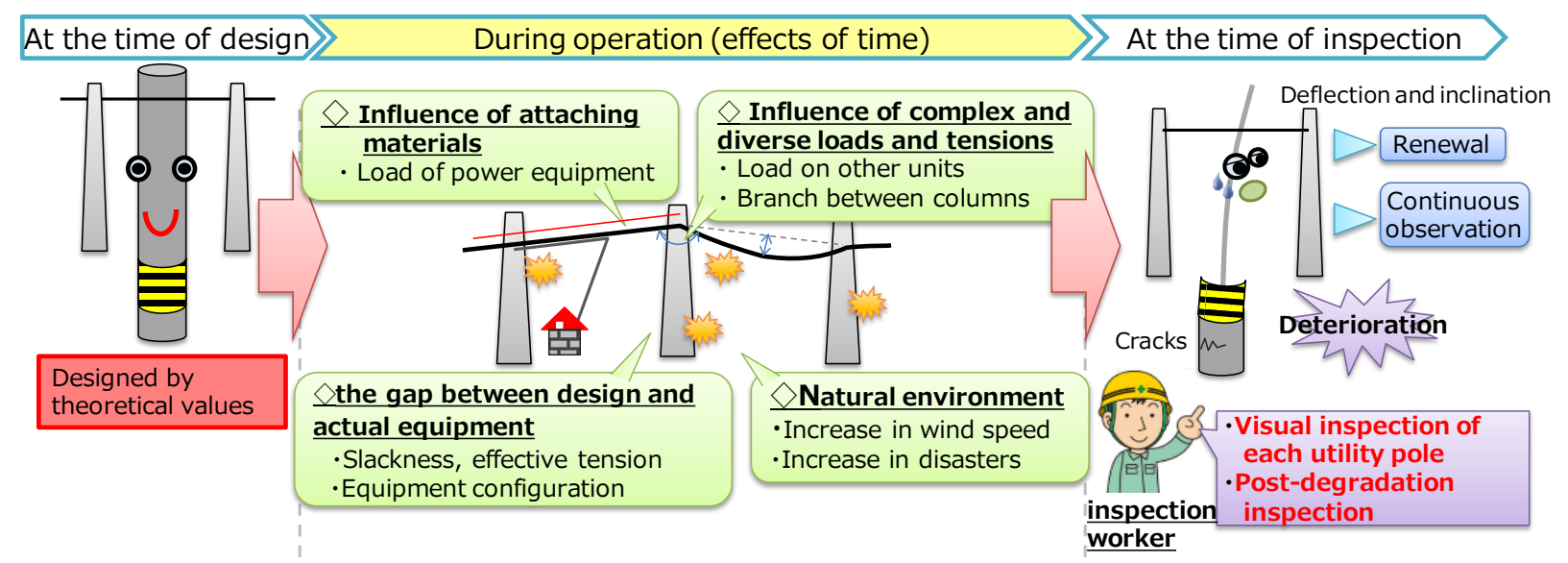

Fig. 2 The process of structural deterioration

In order to achieve these goals, we started two research projects: (i) Load visualization technique, (ii) Relationship between unbalanced loads and structural deterioration. In this paper, we describe the former.

\section{(i) Load visualization technique}

In order to visualize the structural loads carried by outside facilities, we need to calculate the force transmitted from the cable attached to the utility pole (horizontal tension) and the load of other attached materials, and to calculate the moments carried by the utility pole. To achieve this, a 3D point cloud data are acquired by a mobile or fixed laser scanner as shown in Fig. 3. These are used in order to accurately determine the positions at which the horizontal loads are generated. Since the $3 \mathrm{D}$ point cloud can use the data acquired by the above technique [2-4] as it is, no new acquisition work is required.
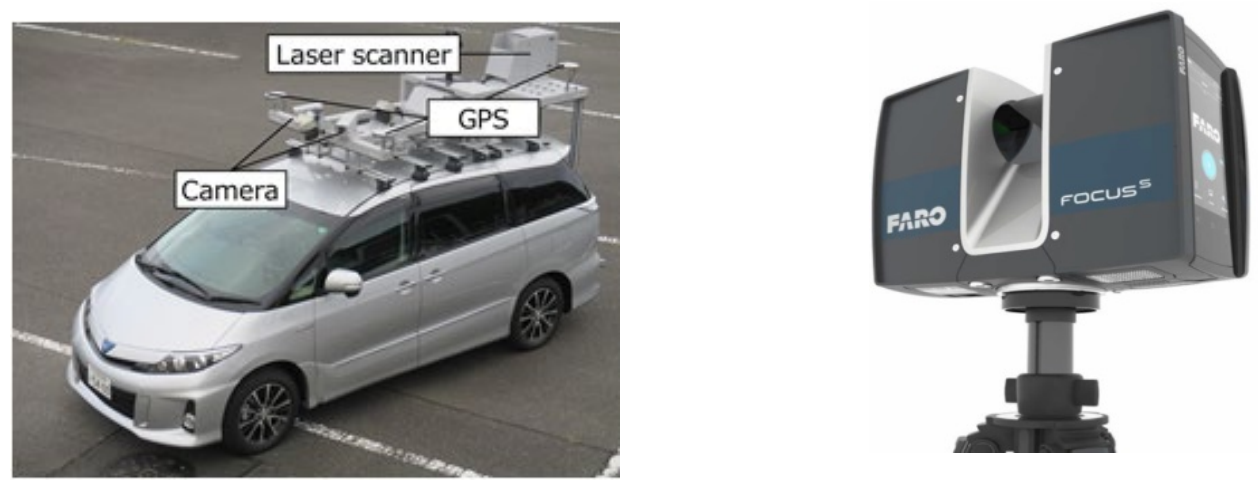

Fig. 3 Mobile and Fixed Laser Scanners

A typical 3D point cloud acquired by these laser scanners is shown in fig. 4. From the figure, it can be seen that there are several cables between the two utility poles and that trees, buildings, etc. have also been acquired as point clouds (Top of Fig. 4). We extract only the necessary information, that is, a point cloud of utility poles and cables (middle section). Next, in order to obtain the horizontal tension from the positional relation between utility poles and the slack of the cable, the catenary function representing the shape of the cable is calculated by the following approximate formula (Lower section). 

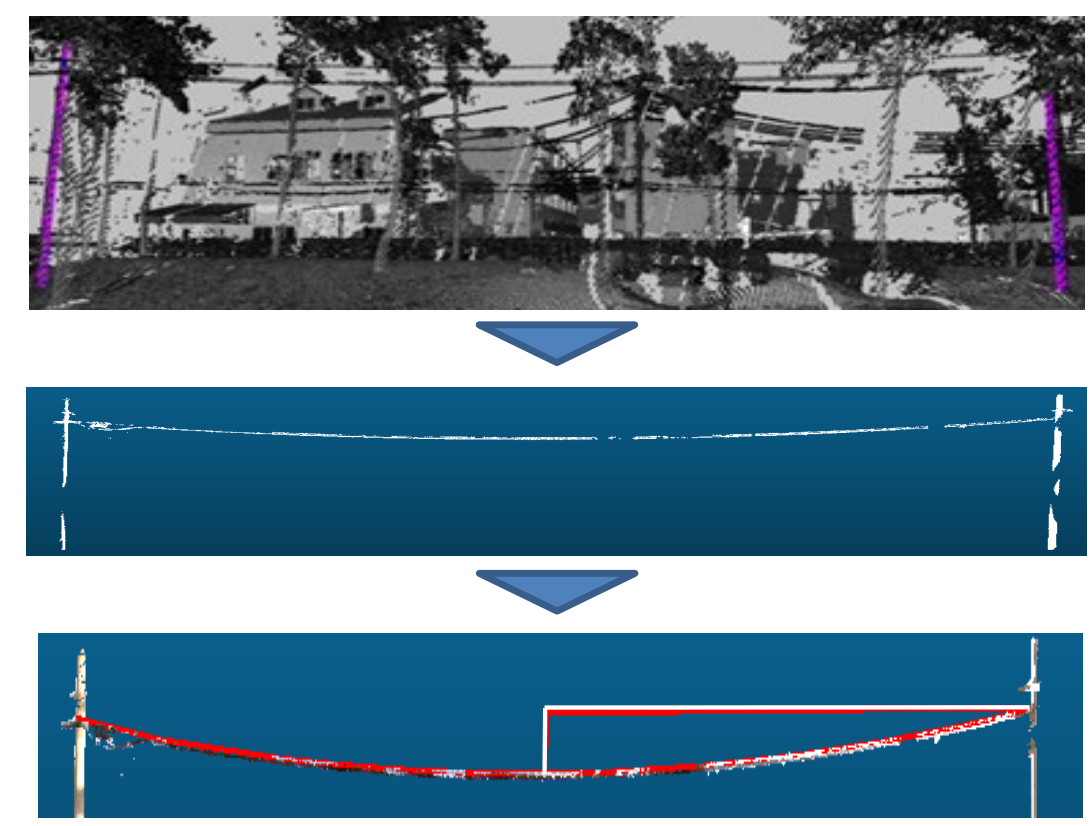

Fig. 4 Method for calculating horizontal tension of cable using 3D point cloud

$$
T=\frac{W s^{2}}{8 d}
$$

Here, $T$ : horizontal tension of the cable, $W$ : cable weight per unit length, $S$ : distance between utility poles, and $d$ : cable slack.

The accuracy of the horizontal tension estimations was examined using the experimental setup shown in Fig. 5.

As shown in the figure, a load cell for measuring tension in wire direction was installed at the end of a cable laid between utility poles separated by $S$, and the horizontal tension was changed by changing slack value $d$. A fixed laser scanner was used to measure the slackness.

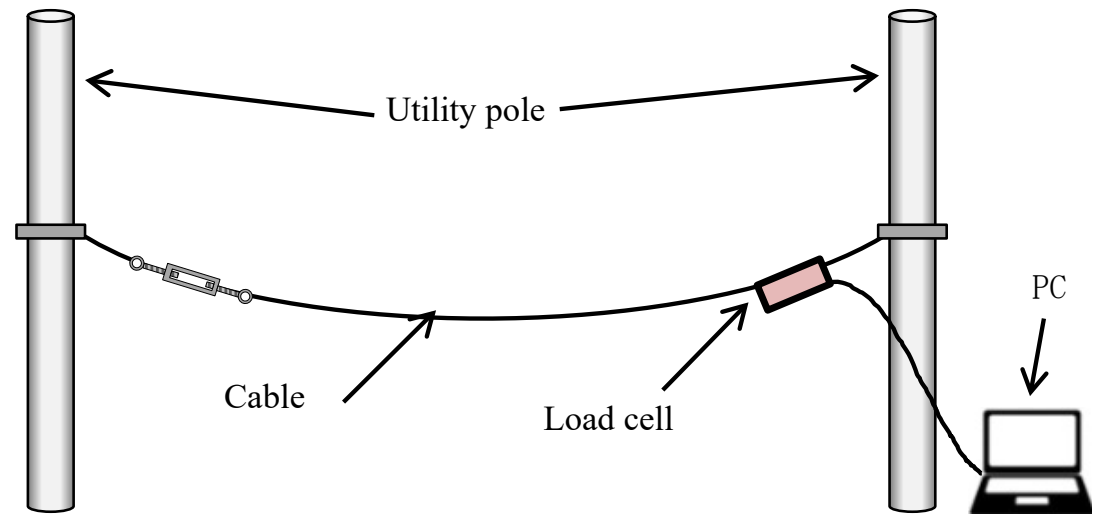

Fig. 5 Experimental setup 
Figure 6 compares measured and estimated loads (determined using equation (1)). In the experiment, the slack value was varied from $0.2 \sim 0.8 \mathrm{~m}$, and the calculated value calculated by equation (1) was compared with the measured value yielded by the technique of Fig. 3 . The measurement by the laser scanner was carried out 30 times, and there were respectively shown average values with maximum and minimum value.

The figure confirms good agreement between the measured and calculated values. The very low error of just $3.6 \%$ conforms the usefulness of this visualization technique.

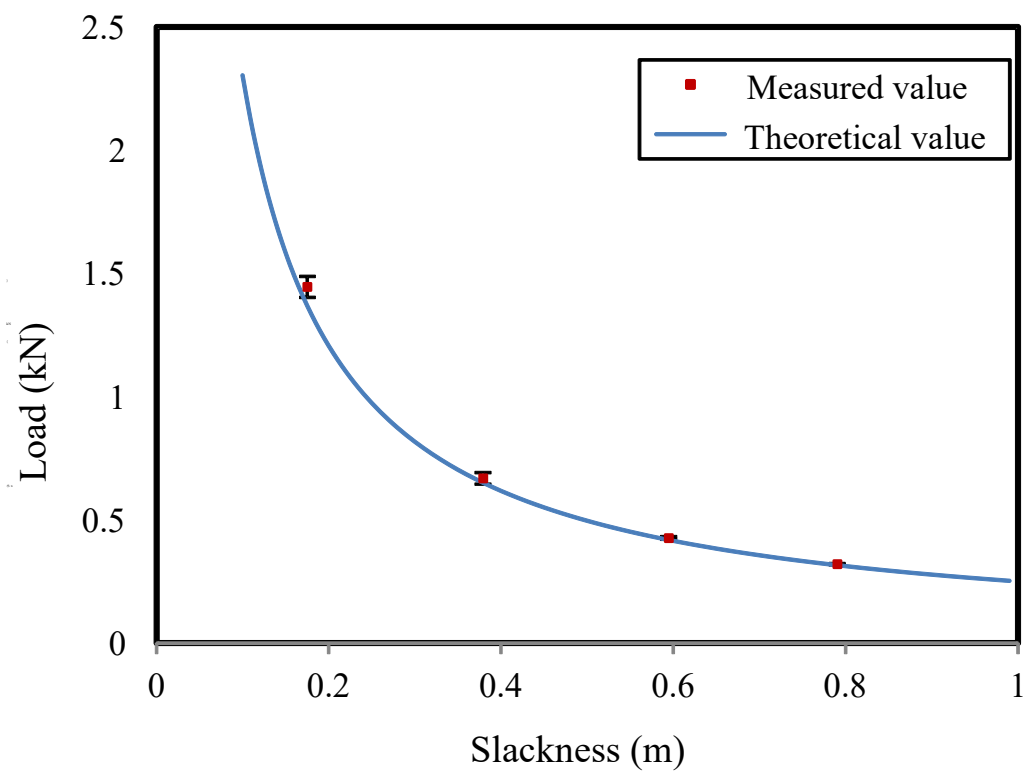

Fig. 6 Comparison of measured and calculated values

When this technology is put into practical use, the utility poles on both sides of the road will be captured by mobile laser scanners. Utility poles that cannot by observed from a road can be covered by fixed laser scanners. This will greatly improve inspection efficiency, and significant cost reductions by the elimination of most manual inspection be expected.

As shown in Fig. 7, a program was developed to obtain the information necessary for load estimation from the point groups, display the positions of the utility poles and cables on a 2D map, and visualize the loasd applied to the utility poles in 3D.

As a result, the inspection worker can quantitatively and accurately understand the loads applied to the utility pole. By countering excessive loads, the structural deterioration can be suppressed, and so realize the long-term safe use of outside facilities.

\section{Conclusion}

This paper described an efficient inspection method for realizing the long-term safe utilization of facilities. It automates the determination of loads placed on utility poles and so allows outside facilities to be maintained in "healthy condition" without structural risk.

The proposal gathers point cloud data of cables and poles, processes the data to determine sides loads, and offers an effective map-based visual interface to assist in corrective work.

In the future, we plan to examine the applicability of this method to actual equipment using laser scanners and software. 


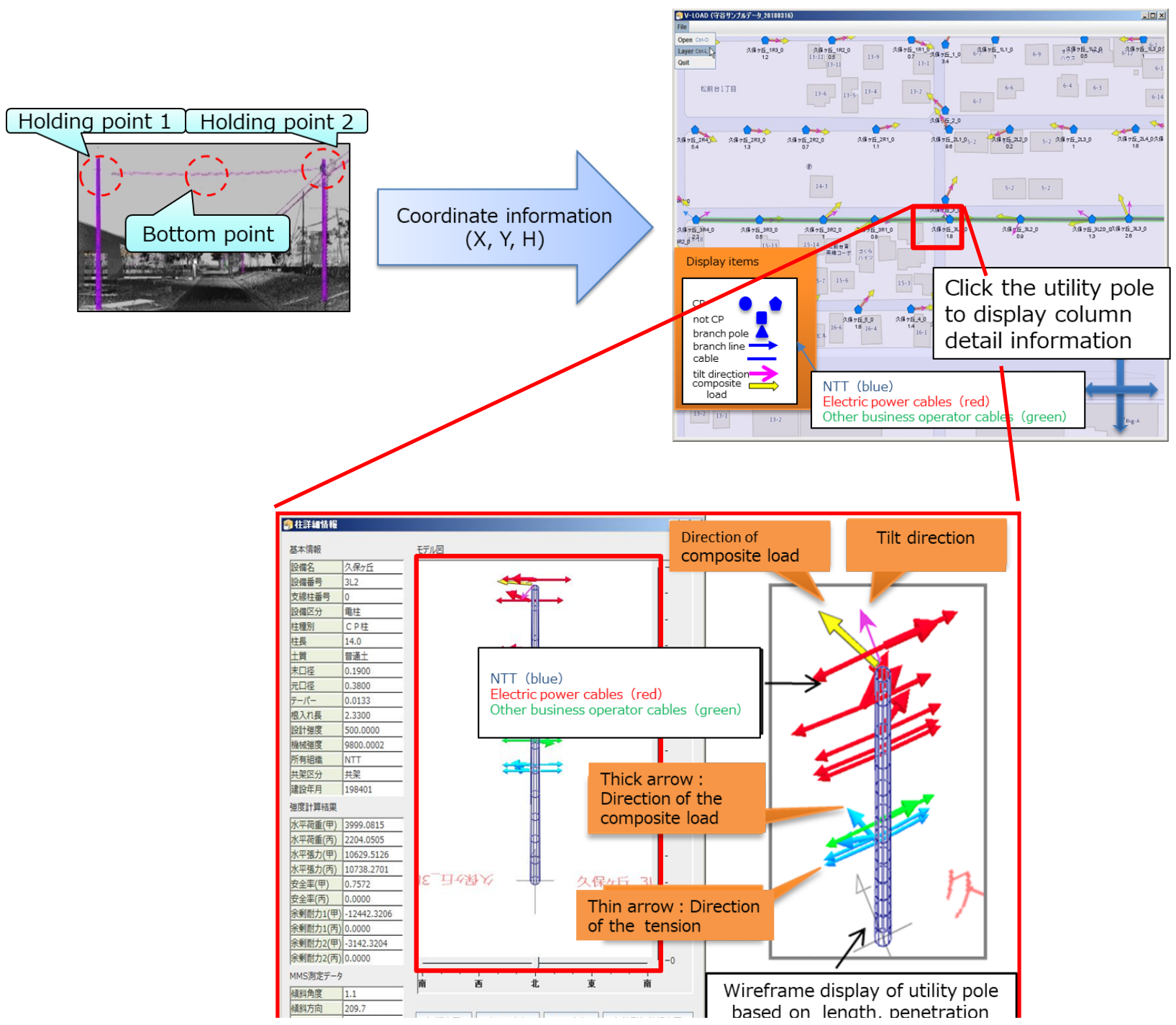

Fig. 7 Software to visualize loads based on this technology

\section{References}

[1] Information NTT East: Telecommunication Facilities, Information NTT East $<$ https://www.ntt-east.co.jp/databook/pdf/2019_allpage.pdf $>$, (Retrieved 27.7.2020)

[2] T. Kurashima, H. Ieda, and M. Waki: Three-dimensional off-site access facility management technology using a mobile mapping system, 3rd Report, Shin-Gaku Gijutsuho, OFT2015-6, pp. 25-30, 2015.

[3] M. Waki, T. Goto, K. Katayama, and T. Kurashima: A study for the application of three-dimensional facility management technology to maintenance and inspection work Three-dimensional off-site access facility management technology using mobile mapping system, 7th Report, Shin-Gaku Gijutsuho, OFT2016-22, pp. 25-28, 2016.

[4] M. Waki, T. Goto, and K. Katayama: 3D facility management technology using a mobile mapping system (MMS), Communications Society Magazine of the Institute of Electronics, Information and Communication Engineers, vol. 12, no. 1, pp. 39-45, 2018. https://doi.org/10.1587/bplus.12.39 